\title{
1 A polygenic architecture with conditionally neutral effects underlies 2 ecological differentiation in Silene
}

4 Susanne Gramlich ${ }^{1}$, Xiaodong Liu ${ }^{1,2}$, Adrien Favre ${ }^{3}$, C. Alex Buerkle ${ }^{4}$ and Sophie

5 Karrenberg $^{1^{*}}$

$6{ }^{1}$ Department of Ecology and Genetics, Uppsala University, Norbyvägen 18D, 75267

7 Uppsala, Sweden

$8{ }^{2}$ The Bioinformatics Center, Department of Biology, University of Copenhagen, Ole

9 Maaløes Vej 5, 2200, Copenhagen, Denmark

$10{ }^{3}$ Senckenberg Research Institute and Natural History Museum, Senckenberganlage 25,

1160325 Frankfurt/Main, Germany

$12{ }^{4}$ Department of Botany, University of Wyoming, 1000 E. University Ave. Laramie, 13 WY82071, USA

$14 *$ corresponding author: Sophie Karrenberg, sophie.karrenberg@ebc.uu.se, +46 (0)76 $15 \quad 8207578$

\section{Word count}

Summary: 196 words

Total (main text): 5569 words

Introduction: 933 words

Materials and Methods: 1820 words

Results: 1026 words

Discussion: 1679 words

Number of Figures: 5 (all in color)

27 Number of Tables: 1

28 Number Supporting Information files: 2 (Figures S1-S8, Tables S1-S4) 


\section{Summary}

52 Key words: adaptation, Bayesian Sparse Linear Mixed Models (BSLMM), conditional 53 neutrality, ddRAD-Seq, reproductive isolation, speciation, Silene

- Ecological differentiation can drive speciation but it is unclear how the genetic architecture of habitat-dependent fitness contributes to lineage divergence. We investigated the genetic architecture of cumulative flowering, a fitness component, in second-generation hybrids between Silene dioica and S. latifolia transplanted into the natural habitat of each species.

- We used reduced-representation sequencing and Bayesian Sparse Linear Mixed Models (BSLMMs) to analyze the genetic control of cumulative flowering in each habitat.

- Our results point to a polygenic architecture of cumulative flowering. Allelic effects were mostly beneficial or deleterious in one habitat and neutral in the other. The direction of allelic effects was associated with allele frequency differences between the species: positive-effect alleles were often derived from the native species, whereas negative-effect alleles, at other loci, tended to originate from the non-native species.

- We conclude that ecological differentiation is governed and maintained by many loci with small, conditionally neutral effects. Conditional neutrality may result from differences in selection targets in the two habitats and provides hidden variation upon which selection can act. Polygenic architectures of adaptive differentiation are expected to be transient during lineage divergence and may therefore be unrelated to high genetic differentiation at the underlying loci.

51 


\section{Introduction}

55 Adaptation to different habitats can promote divergence and speciation and can mediate 56 population persistence under changing conditions (Nosil, 2012; Savolainen et al., 2013).

57 Evolutionary trajectories toward adaptive differentiation depend on the number and 58 effect sizes of loci controlling fitness, on allelic effects of such loci in alternative 59 habitats and on gene flow (Savolainen et al., 2013; Tigano \& Friesen, 2016; Kokko et 60 al., 2017). However, despite recent progress in the theoretical understanding of 61 adaptation (Yeaman, 2015; Mee \& Yeaman, 2019; Booker et al., 2021), empirical 62 studies on the genetic control of fitness in natural habitats is still scarce (Wadgymar et $63 a l ., 2017)$. Here we provide such a study using two ecologically differentiated but 64 hybridizing campions (Silene).

65 Adaptive differentiation is often depicted as a classic cartoon with populations outperforming other populations in their home habitat but not in the foreign habitat (Kawecki \& Ebert, 2004). A similar pattern has been proposed to operate at the genetic level: alleles with positive effects on fitness in one habitat have negative effects in the alternative habitat, termed antagonistic pleiotropy (Anderson et al., 2011; Savolainen et al., 2013). Empirical data, however, suggests that such antagonistic pleiotropy is rare in natural populations, although this could partially be caused by limited statistical power (Anderson et al., 2011; Anderson et al., 2014; Wadgymar et al., 2017). An alternative pattern, conditional neutrality, where alleles have neutral effects in one environment and are under positive or negative selection in the other, may be much more prevalent (Anderson et al., 2011; Savolainen et al., 2013; Wadgymar et al., 2017). In fact, even at the phenotypic level, populations often perform differently in one habitat but not in the other (Leimu \& Fischer, 2008). Importantly, loci with antagonistic pleiotropy for fitness in alternative habitats are expected to be maintained by natural selection and contribute to population differentiation even with high gene flow, whereas conditionally neutral loci can only lead to transient allele frequency differences in high-gene flow scenarios (Mitchell-Olds et al., 2007; Savolainen et al., 2013; Tiffin \& Ross-Ibarra, 2014; Mee \& Yeaman, 2019; Booker et al., 2021). If adaptive allele frequency changes are predominantly transient, even in the presence of consistent selection, prospects will be poor for finding loci underlying adaptive differentiation with commonly used 
87 A further major determinant of adaptive differentiation concerns the number and effect 88 sizes of the underlying loci. In general, the distribution of effect sizes for complex traits 89 (including fitness) is expected to be exponential (Orr, 1998, 2005) such that adaptation 90 is mainly due to many loci with small effects, while large-effect loci, although not generally unimportant, are rare (Orr, 1998; Orr, 2005; Rockman, 2012; Savolainen et al., 2013; Boyle et al., 2017; Selby \& Willis, 2018). A large number of segregating loci underlying adaptation further promotes transient genetic architectures of adaptation (Yeaman \& Whitlock, 2011; Yeaman, 2015; Booker et al., 2021). Polygenic or even omnigenic genetic architectures, involving most of the genome, render identification of all individual loci both practically impossible and undesirable (Rockman, 2012). As effect sizes decrease and the number of loci increases, it becomes progressively more difficult to detect individual genotype-phenotype associations and to distinguish them from spurious associations due to linkage. For this reason, promising methods, such as Bayesian Sparse Linear Mixed Models (BSLMMs), identify genotype-phenotype associations of all loci simultaneously rather than individually, assume polygenic genetic architectures and remove effects that can be attributed to linkage disequilibrium (Zhou et al., 2013; Gompert et al., 2017). The approach has been successfully applied in studies on adaptive divergence, for example in pine (Lind et al., 2017) and in Arabidopsis (Exposito-Alonso et al., 2019) .

In this study, we investigate the genetic basis of differential adaptation in two dioecious sister species of Silene (Caryophyllaceae), Silene dioica (L.) Clairv. and S. latifolia Poiret. Demographic models indicate that the two species diverged with gene flow within the last 120000 years reaching a neutral sequence divergence ( $D_{a}$, autosomes) of 0.0027 and genetic differentiation, $\mathrm{F}_{\mathrm{ST}}$, of 0.28 (Hu \& Filatov, 2015; Liu et al., 2020). The pink-flowered S. dioica occurs in moister and colder habitats like meadows, pastures or forests and occupies a wide range of elevations up to more than $2300 \mathrm{~m}$ a. s. 1., while the white-flowered S. latifolia is found on drier, warmer and more disturbed sites like dry meadows, arable fields or road sites at elevations up to $1000 \mathrm{~m} \mathrm{a}$. s. 1 .

115 (Friedrich, 1979; Karrenberg \& Favre, 2008). Ecological differentiation and assortative pollination constitute strong barriers to gene flow in this species pair, but the two species are still fully cross-fertile (Goulson \& Jerrim, 1997; Karrenberg et al., 2019). of traits associated with reproductive isolation, such as flower color, flowering 
120 phenology, first-year flowering and specific leaf area in this system (Liu \& Karrenberg, 121 2018).

122 Here we investigated the genetic architecture of adaptation using recombinant second-

123 generation hybrids $\left(\mathrm{F}_{2}\right)$ from a multi-site field transplant experiment where the two

124 species exhibited strong evidence of habitat adaptation: each species outperformed the 125 other in its own habitat in terms of flowering and survival over four years (Fig. 1, Favre 126 et al., 2017). We focused on the following questions: (1) What is the genetic 127 architecture underlying differential habitat adaptation? (2) Is antagonistic pleiotropy or 128 conditional neutrality the predominant pattern when comparing allelic effects across 129 habitats? and (3) Are fitness effects associated with allele frequency differences 130 between the two species; for example, are beneficial alleles more likely to be derived 131 from the native species?

\section{Materials and Methods}

\section{Transplant experiment, measurements and sampling}

134 Second-generation $\left(\mathrm{F}_{2}\right)$ hybrids between Silene dioica (L.) Clairv. and Silene latifolia 135 Poir., derived from $36 \mathrm{~F}_{0}$ individuals of three populations of each species, were 136 transplanted as juveniles into four natural sites, two in each species's habitat, as part of a 137 larger experiment with six sites (Favre et al., 2017, Supporting Information Tables S1, $138 \mathrm{~S} 2$, and S3). Sites of S. dioica were situated at higher altitudes with a colder climate 139 and shorter growing season as compared to the S. latifolia sites (Supporting Information 140 Table S1). Leaf samples were collected prior to transplantation and silica-dried and we 141 selected four of the six sites for this study based on availability and quality of leaf 142 samples. Flowering and survival were assessed over four years and cumulative 143 flowering was calculated as the number of times an individual flowered plus 1 if it 144 survived to the end of the experiment, for detailed analyses of survival and flowering 145 see Favre et al. (2017). We sampled 4-6 $F_{2}$ individuals from each of $18 F_{2}$ families at 146 each of the four selected sites ( $298 \mathrm{~F}_{2}$ individuals in total), striving to include both high 147 and low fitness individuals from each family and site. To assess allele frequency in the 148 two species we further included samples of $32 \mathrm{~F}_{0}$ individuals and used existing data for 149 the remaining four $\mathrm{F}_{0}$ individuals (Liu \& Karrenberg, 2018, Supporting Information 150 Table S3). 
151 For illustration purposes (Fig. 1), we re-analyzed cumulative flowering in the two

152 species and their first- and second-generation hybrids for the four sites used here, (18-36

153 families per cross type, 5-20 individuals per family, 5 blocks per site, Favre et al. 2017,

154 Supporting Information Table S2). We used linear mixed models of cumulative

155 flowering in each habitat with cross type as a fixed factor and family and block nested

156 in site as random factors using the lme4 package (Bates et al., 2015) for $\mathrm{R}$ version 4.02

157 (R Core Team, 2020). We extracted least square means of cumulative flowering and

158 performed multiple comparisons between cross types within sites with Holm correction

159 of P-values in the emmeans package (Lenth, 2020). Cumulative flowering was

$160 \log (\mathrm{Y}+1)$-transformed to yield normally distributed residuals and improve model fit.

161 Means and standard errors are reported back-transformed to the original scale.

DNA extraction and sequencing

163 We extracted genomic DNA from silica-dried leaf tissue with Qiagen's DNeasy Plant 164 Mini Kit (Qiagen, Germany) and quantified DNA using a Qubit dsDNA HS fluorometer 165 (Life Technologies, Sweden). Double-digest RAD sequencing (ddRAD-seq) libraries 166 were prepared with EcoRI and Taq $\alpha$ I restriction enzymes as described in Liu and 167 Karrenberg (2018). After enzymatic digestion, DNA fragments were ligated with 168 barcoded adaptors and size-selected to approximately $550 \mathrm{bp}$ (Peterson et al., 2012). In 169 total, eight 48-plex libraries were sequenced on an Illumina HiSeq 2500 system at the 170 SNP\&SEQ technology platform of SciLifeLab, Uppsala, Sweden using 125-bp paired171 end chemistry and two libraries per lane. $\mathrm{F}_{0}$ individuals were included in 2 libraries to 172 achieve higher coverage.

\section{Bioinformatic analysis - processing of raw reads and variant filtering}

174 The total sequencing output was 1,382,838,294 reads for $298 \mathrm{~F}_{2}$ individuals (mean with 175 one standard error: 4,656,021 $\pm 233,639$ reads) and 461,127,142 reads for $32 \mathrm{~F}_{0}$ 176 individuals (mean: 14,410,223 $\pm 1,093,409$ reads); data on the remaining four $\mathrm{F}_{0}$ 177 individuals (Supplementary Information Table S3) was available from (Liu \& 178 Karrenberg, 2018). We processed the ddRAD-sequence reads following the dDocent 179 pipeline (Puritz et al., 2014). After de-multiplexing of raw reads using STACKS 2.0b 180 (Catchen et al., 2013) and trimming with fastp (Chen et al., 2018), we used BWA MEM 181 0.7.17 with default parameters ( $\mathrm{Li}, 2013)$ to map reads to ddRAD-seq-generated 
182 reference contigs, which were previously assembled from eight deeply sequenced

183 individuals of both species and hybrids and corresponded to 95,040,562 bp in total, 184 corresponding to approximately $3.4 \%$ of the S. latifolia genome (Liu \& Karrenberg, 185 2018; Liu et al., 2020). Only a partial genome sequence (one third of the $2.8 \mathrm{Gbp}$ 186 genome) with short scaffolds $\left(\mathrm{N}_{50}=10,785 \mathrm{bp}\right)$ is currently available for S. latifolia 187 (Krasovec et al., 2018).

Variants were called with FreeBayes 1.1.0 (Garrison \& Marth, 2012) without population priors using the following parameters: minimum mapping quality 30, minimum base quality 20 , maximum complex gap 3 , minimum repeat entropy 1 , binominal-obs-priors 1, and use-best-n-alleles 10. Variants were filtered following O'Leary et al. (2018): First, VCFtools 0.1.15 (Danecek et al., 2011) was used to retain SNP sites with a minimum depth of 3, quality of 30, mean depth of 10 and allele count of 3. Secondly, we used Vcffilter implemented in vcflib/2017-04-04 (https://github.com/vcflib/vcflib) to retain sites with an allele balance either between 0.25 and 0.75 or lower than 0.01 , a quality/depth ratio of $>0.25$, and a mapping quality ratio between 0.9 and 1.05. We further used Vcffilter to remove loci with differences in read pairing between the alleles or with or excessive read depths (parameters as suggested in O'Leary et al., 2018). We reduced the dataset to bi-allelic sites and removed $6 \mathrm{~F}_{2}$ individuals with more than $99 \%$ missing data. SNPs in perfect linkage disequilibrium $\left(\mathrm{r}^{2}=1\right)$ within the $\mathrm{F}_{2}$ individuals were removed using PLINK 1.9 (Purcell et al., 2007, http://pngu.mgh.harvard.edu/purcell/plink/ ). The filtered dataset contained $290 \mathrm{~F}_{2}$ individuals (S. dioica-habitat: 134, S. latifolia-habitat: 156) and 89'524 loci. Of these, 42'090 loci with both alleles in both habitats and genotypes for more than $95 \mathrm{~F}_{2}$ individuals in each habitat were used for further analyses. These loci had an average read depth of $15.02 \pm 0.03$ (median 13.83) for the $F_{2}$ individuals, $36.85 \pm 0.08$ (median: 33.09) for the $32 \mathrm{~F}_{0}$ individuals sequenced in this study and $41.39 \pm 0.17$ (median: 36.25) for the $4 \mathrm{~F}_{0}$ individuals from Liu and Karrenberg (2018). We used genotype probabilities ranging from 0 to 2 , calculated from genotype likelihoods (https:// github.com/ visoca/popgenomworkshop-

211 gwas_gemma/tree/master/scripts/bcf2bbgeno.pl, accessed 28 January 2019), rather than 212 called genotypes (Nielsen et al., 2011). Genotype probabilities of 0 and 2 denote 213 homozygosity for the reference and alternative allele, respectively, while 1 indicates 214 heterozygosity. 
215

216

217

218

219

220

221

222

223

224

225

226

227

228

\section{Genetic structure}

We checked for genetic structure in the $F_{2}$ individuals using Principal Components Analysis (PCA) with the ade4 package (Dray and Dufour, 2007), based on genotype probabilities. For this analysis we reduced the dataset to 220 SNP loci with data available in all $290 \mathrm{~F}_{2}$ individuals used in the genetic association analysis. Results did not differ from analyses on more or all loci with missing values replaced by average genotype probabilities.

\section{Genetic association analysis}

We used Bayesian Sparse Linear Mixed Models (BSLMM) in GEMMA 0.98.1 (Zhou et al., 2013) to investigate the genetic architecture of cumulative flowering. BSLMMs are a combination of linear mixed models, which assume that every variant has an effect, and Bayesian Variable Selection Regression, which assumes that only a small proportion of the variants has an effect (Zhou et al., 2013). BSLMM analyses were performed separately for each habitat using cumulative flowering values standardized within sites and genotype probabilities. A centered relatedness matrix was used as a covariate to take account of the family structure and the wide cross, according to the standard BSLMM method implemented in GEMMA (Zhou et al., 2013).

We characterized genetic architectures using estimates of the following hyperparameters: the proportion of phenotypic variance explained by all SNPs in the model ( $P V E)$, the proportion of $P V E$ explained by SNPs with nonzero effects $(P G E)$, and the number of SNPs with a measurable effect on the phenotype $(n-\gamma)$. In studies with incomplete genome coverage such as this one, $P V E$ can be interpreted as broad sense heritability $H^{2}$, whereas the product of $P V E$ and $P G E$ can be interpreted as narrow-sense heritability $h^{2}$ (Zhou et al., 2013; Gompert et al., 2017; Bresadola et al., 2019). For each SNP locus, we estimated the posterior inclusion probability (PIP, the proportion of iterations in which a SNP had a nonzero effect on phenotypic variation) and the effect size of the alternative allele on cumulative flowering. We report both raw effect estimates $(\hat{\beta})$, the effect of a locus on the phenotype when it is included in the model, and model-averaged effect estimates $(\bar{\beta})$ that take into account the posterior inclusion probability of a locus in the models (Zhou et al., 2013; Gompert et al., 2017). BSLMMs were run five times with 10,000,000 burn-in steps and 40,000,000 iterations with a thinning interval of 10 . Convergence of the five runs was checked graphically. 
247 Hyperparameters were estimated after combining posterior distributions across runs;

$248 P I P, \hat{\beta}$ and $\bar{\beta}$ (per locus) were averaged across runs. A threshold of $P I P>0.01$ was

249 used to identify SNPs with sparse effects (Gompert et al., 2013; Comeault et al., 2014).

\section{Allelic effects and allele frequencies}

251 We graphically evaluated whether alleles have universal effects or display antagonistic

252 pleiotropy or conditional neutrality by plotting per-locus effect sizes ( $\hat{\beta}$ and $\bar{\beta}$ ) in the

$253 S$. dioica habitat against effect sizes in the $S$. latifolia habitat. Note that $\beta$-values reflect

254 effect estimates of the alternative allele replacing the reference allele. The de novo

255 ddRADseq reference sequences used here contain alleles derived from both species (Liu

256 \& Karrenberg, 2018; Liu et al., 2020) and do not inform on allelic origin.

257 To analyze whether allelic origin is associated with fitness effects, we calculated the 258 frequency of the alternative allele $\left(\mathrm{AF}_{\text {alt }}\right)$ in the $18 \mathrm{~F}_{0}$ individuals of each species (Tables $259 \mathrm{~S} 2$ and $\mathrm{S} 3$ ) for loci that had genotypes for $>12 \mathrm{~F}_{0}$ individuals per species at individual 260 read depths of at least $6\left(36,161\right.$ loci). For each SNP locus, we expressed $\mathrm{AF}_{\text {alt }}$ as the 261 average genotype probability per species divided by 2 . We further estimated the allele 262 frequency difference between species $\left(\mathrm{AFD}_{\text {alt }}\right)$ as $\mathrm{AF}_{\text {alt }}($ S. dioica $)-\mathrm{AF}_{\text {alt }}($ S. latifolia $)$. $263 \mathrm{AFD}_{\text {alt }}$ values thus range from -1 (alternative allele fixed in S. latifolia $\mathrm{F}_{0}$ individuals, 264 and reference allele fixed in $S$. dioica $\mathrm{F}_{0}$ individuals) to 1 (alternative allele fixed in $S$. 265 dioica $\mathrm{F}_{0}$ individuals, and reference allele fixed in $S$. latifolia $\mathrm{F}_{0}$ individuals). We tested 266 whether $\mathrm{AFD}_{\text {alt }}$ differs between loci with positive and negative effects on cumulative 267 flowering in each habitat using a general permutation test in the $\mathrm{R}$ package coin 268 (Hothorn et al., 2008).

\section{Method validation}

270 We assessed the power of BSLMMs to detect genotype - phenotype associations in our 271 data using a simulation approach similar to that in Gompert et al. (2017). Phenotypes 272 were simulated on the basis of the observed genetic data in 290 individuals for four 273 combinations of heritability $\left(h^{2}=0.2\right.$ or $\left.h^{2}=0.05\right)$ and number of functional variants $274(N=10$ or $N=50)$. We simulated a normally distributed trait for which nine 275 individuals of each family were randomly drawn. We reduced the simulated dataset to 276150 individuals at random to match the sample size in our data. For each combination 277 of $h^{2}$ and n 30 sets of simulated phenotypes were used as input for BSLMMs with 
$27810,000,000$ burn-in steps and 40,000,000 iterations. For each simulation, PVE, PGE

279 and $n-\gamma$ were estimated as well as the correlation between the simulated effect size and

280 effect size estimates $(\hat{\beta}$ and $\bar{\beta})$.

282 Results

283 Cumulative flowering and genetic structure

284 Each species outperformed the other in its own habitat and performed best in its habitat

285 in terms of cumulative flowering, providing strong evidence for habitat adaptation (Fig.

286 1, Favre et al., 2017). $\mathrm{F}_{1}$ hybrids were intermediate for cumulative flowering and $\mathrm{F}_{2}$

287 hybrids flowered less often than $F_{1}$ hybrids on average (Fig. 1, Favre et al., 2017).

288 The $\mathrm{F}_{2}$ families exhibited extensive genetic variation in the PCA, with clustering of 289 individuals within families but without any other emerging patterns (Supplementary 290 Information Figs. S1 and S2). For most loci, the alternative allele occurred at low 291 frequency in one or both species, but all combinations of allele frequencies between 292 species were found in the data, including loci with high differentiation where the 293 alternative allele was at high frequency in one species and at the same time near absent 294 from the other species (Fig. 2).

\section{Association analysis}

296 The proportion of phenotypic variation explained (PVE) by BSLMMs was 0.12 and 2970.10 for the S. dioica and the S. latifolia habitat, respectively (medians of posterior 298 distributions, Table 1, posterior distributions are shown in the Supporting Information 299 Figs. S3 and S4). Less than half of the PVE could be attributed to sparse effects, 300 leading to estimates of narrow-sense heritability $\left(h^{2} ; P G E \times P V E\right)$ of 0.05 and 0.03 for 301 the S. dioica and S. latifolia habitat (Table 1). The number of loci with non-zero effects $302(n-\gamma)$ was estimated to be 11 in the S. dioica habitat and 16 in the S. latifolia habitat 303 (Table 1). Median PIP for individual SNPs was 0.001 in both habitats and we detected 3043 loci with PIP $>0.01$, one in the S. dioica habitat and two in the S. latifolia habitat 305 (Supporting information Table S4). PIP for individual SNPs could not be summed over 306 genomic windows because no continuous reference genome is available (Krasovec et 
307

308

309

310

311

al., 2018) and we used a ddRADseq-generated de novo reference in this study (Liu \& Karrenberg, 2018; Liu et al., 2020).

\section{Allele specific effects in alternative habitats and origin of alleles}

Raw effect size estimates $\hat{\beta}$ for cumulative flowering (in units of standard deviations, i.e., z-scores) ranged from -0.57 to 0.28 in the $S$. dioica habitat and from -0.52 to 0.34 in the $S$. latifolia habitat. Positive or negative allelic effects in one habitat $(\hat{\beta}>>0$ or $\hat{\beta}<<$ $0)$ were associated with very low $\hat{\beta}$ values $(\hat{\beta} \approx 0)$ in the other habitat, consistent with conditional neutrality (Fig. 3, cross pattern). A single locus showed opposing effects in the two habitats, $\hat{\beta} \ll 0$ in the $S$. dioica habitat and $\hat{\beta} \gg>0$ in the S. latifolia habitat, suggestive of antagonistic pleiotropy (Fig. 3). Analyses for model-averaged effect sizes, $\bar{\beta}$, exhibited a very similar pattern (Supporting information Fig. S5), but with much smaller values due to multiplication with low posterior inclusion probabilities.

A striking pattern in our data is that alleles with effects on cumulative flowering were significantly associated with higher allele frequencies in $\mathrm{F}_{0}$ individuals of the native species whereas alleles with negative effects on cumulative flowering were significantly more likely to be derived from the non-native species (Fig. 3, Supporting Information Figs. S5, S6 and S7). In the S. dioica habitat, loci with positive effects of the alternative allele on cumulative flowering had a positive median $\mathrm{AFD}_{\text {alt }}$ (alternative allele more common in S. dioica, red on Fig. 3), that were significantly different from the negative median $\mathrm{AFD}_{\text {alt }}$ (alternative allele more common in S. latifolia, blue on Fig. 3) at negative-effect alleles (Supporting Information Figs. S6 and S7). In the S. latifolia habitat, we observed the reverse pattern: loci with positive effects had negative median $\mathrm{AFD}_{\text {alt }}$ values that differed significantly from the positive median $\mathrm{AFD}_{\text {alt }}$ values for negative-effect loci (Supporting information Figs. S6 and S7). These patterns were observed for both $\hat{\beta}$ and $\bar{\beta}$, when using a subset of the approximately $0.5 \%$ loci with strongest effect sizes (absolute values of $\hat{\beta}$ over 0.1 and absolute values of $\bar{\beta}$ over 0.003 ) and when using all loci (i.e., negative-effect loci with $\hat{\beta}$ or $\bar{\beta}>0$ and positiveeffect loci with $\hat{\beta}$ or $\bar{\beta}<0$, Supporting Information Figs. S6 and S7). 
336 Alleles with positive effects on cumulative flowering in each habitat $(\hat{\beta}>0.1)$ were rare

337 in the foreign species but at appreciable frequencies in the native species; this effect was 338 stronger in the S. latifolia habitat than in the S. dioica habitat (Fig. 4). Loci with 339 negative effects $(\hat{\beta}<-0.1)$, in contrast, were rare in the native species but at moderate 340 or high frequencies in the foreign species (Fig. 4). These allele frequency patterns 341 across species differ strongly from the overall allele joint allele frequency pattern for all 342 loci (Fig. 2c).

\section{Method validation}

344 BSLMM estimates of narrow-sense heritability $\left(h^{2}\right)$ in simulated datasets responded to 345 changes in simulated heritabilities, as expected (Fig. 5). BSLMMs on simulated data 346 with a low $h^{2}$ of 0.05 recovered $h^{2}$ estimates in the correct range, while models on 347 simulated data with a high $h^{2}$ of 0.2 yielded lower than expected $h^{2}$ estimates, especially 348 when the number of sparse-effect loci was high $(\mathrm{N}=50$, Fig. 5). The number of sparse349 effect loci was correctly estimated for simulated datasets with few loci $(\mathrm{N}=10)$, but 350 strongly underestimated when the simulated number of loci was high (Fig. 5). 351 Correlations of simulated and estimated $\hat{\beta}$ and $\bar{\beta}$-values were highest for datasets with 352 high simulated heritabilities and declined for datasets with lower heritability and a 353 higher number of functional loci (Fig. 5, Supporting Information Fig. S8). In 354 simulations with $h^{2}=0.02, \hat{\beta}$ exhibited higher correlations with simulated effects sizes 355 than $\bar{\beta}$ (Supporting Information Fig. S8).

356 These simulations thus show that our analyses can recover heritabilities and general 357 patterns in effect size, however, estimates of the number of sparse effect loci $(n-\gamma)$ must 358 be treated with great caution. The consistency between estimated and simulated $h^{2}$ for 359 datasets with low simulated $h^{2}$ shows that the low $h^{2}$ on our empirical data are reliable, 360 especially as such low $h^{2}$ estimates were very rare in the high $h^{2}$ simulations. We 361 expect that the apparent underestimation of $n-\gamma$ also decreases posterior inclusion 362 probabilities and thus $\bar{\beta}$.

\section{Discussion}

364 Our results suggest that ecological differentiation between the campions Silene dioica 365 and S. latifolia has a polygenic architecture, based on a reciprocal transplant experiment 
366 with recombinant second-generation hybrids between the two species. Many alleles had 367 small effects on cumulative flowering, a fitness component, but only in one of the two 368 habitats, conforming to a conditional neutrality pattern. Despite small effect sizes, the 369 direction of allelic effects was consistent with selection for native alleles and against 370 non-native alleles. This suggests that habitat adaptation is maintained by species371 specific variants and that habitat dependent selection on hybrids reduces the inflow of 372 non-native alleles. Below we discuss the implications of these findings for lineage 373 divergence as well as the advantages and limitations of our approach.

375 Heritability of cumulative flowering, a fitness component, was low to moderate in $\mathrm{F}_{2}$ 376 hybrids between S. dioica and S. latifolia in the habitats of both species. Using 377 Bayesian Sparse Linear Mixed Models (BSLMM) we estimated narrow-sense 378 heritability $h^{2}$ (PVE x $P G E$ ) to be 0.04 and 0.03 and broad-sense heritability $H^{2}(P V E)$ 379 to be 0.12 and 0.10 for S. dioica and S. latifolia habitats, respectively. Our simulations 380 indicate that such low $h^{2}$ values can be recovered fairly well with BSLMM analyses, as 381 also reported by Gompert et al. (2017). Similar low to moderate heritabilities have 382 previously been found for complex traits, for example for height in Pinus albicaulis 383 (Lind et al., 2017) and in Populus (Bresadola et al., 2019) as well as in Arabidopsis 384 thaliana accessions, where heritability for fitness components varied strongly between 385 experimental sites (Exposito-Alonso et al., 2019). Our analyses suggest a polygenic 386 genetic architecture of cumulative flowering in Silene with estimates of the number of 387 sparse-effect loci estimated to 11 in the S. dioica habitat and to 16 in the S. latifolia 388 habitat; however, our simulations indicate that the number of sparse effect loci in our 389 dataset is difficult to estimate with BSLMM analyses, similar to results from Gompert 390 et al. (2017). In many other systems, oligo- or polygenic control of fitness components 391 was detected in natural settings, especially when the number of contributing loci was 392 explicitly modelled (Lind et al., 2017; Bresadola et al., 2019; Exposito-Alonso et al., 393 2019). A polygenic genetic architecture of fitness components in our study system does 394 appear likely given that QTL studies detected many loci distributed throughout the 395 genome that were associated with ecologically relevant traits (Liu \& Karrenberg, 2018; 396 Baena $\square$ Díaz et al., 2019). 
397 Limitations of studies on the genetic architecture of fitness in natural habitats include

398 the number of individuals and sites that can be studied as well as the genetic resolution

399 in the experimental material. Where markers are used, as in our study, most

400 associations will be the result of linkage to causal variants rather than due to causal

401 variants themselves. This reduces estimates of effect sizes and may lead to a situation

402 where multiple loci linked to the same causal variant are included in alternative

403 BSLMM iterations reducing the posterior inclusion probability $(P I P)$ of each linked

404 locus (Bresadola et al., 2019). We used a highly variable multi-cross $\mathrm{F}_{2}$ population with

405 a limited number of recombination events, where markers likely were associated with

406 larger genomic regions derived from each species. Recombination and genetic variation

407 included in our material differs markedly from other studies using largely homozygous

408 selfing accessions (Arabidopsis, Exposito-Alonso et al., 2019), within-species crosses

409 or recombinant inbred lines derived from a small number of individuals (Arabidopsis,

410 Fournier-Level et al., 2013; Leinonen et al., 2013; Ågren et al., 2017) or naturally

411 recombinant wild-collected hybrids (Populus, Bresadola et al., 2019). These

412 differences can make it difficult to compare results and model performance across

413 studies. Despite its limitations, our setup has the advantage that the multi-cross $F_{2}$

414 population used here approximates a natural contact site situation, where early-

415 generation hybrids grow in the habitats of the two species when they come into contact

416 (Karrenberg \& Favre, 2008).

\section{Conditional neutrality is the prevailing pattern}

418 Allelic effects conformed to a conditional neutrality pattern in our study: both positive 419 and negative allelic effects on cumulative flowering in one habitat were associated with 420 near-zero effects in the other habitat; only one locus deviated from this pattern. This

421 finding further strengthens the view that conditional neutrality is more common in 422 natural settings than antagonistic pleiotropy, as has been shown in monkeyflowers (Hall 423 et al., 2010) and Arabidopsis (Fournier-Level et al., 2013; Ågren et al., 2017; Exposito424 Alonso et al., 2019), switchgrass (Lowry et al., 2019) and Lycaeides butterflies 425 (Gompert et al., 2015). However, strong evidence for conditional neutrality is generally 426 difficult to provide, because this would require evidence for the absence of an effect in 427 one of the habitats (Anderson et al., 2014; Mee \& Yeaman, 2019). Interestingly, 428 antagonistic pleiotropy appears to be detected more readily in controlled experimental 429 evolution studies with microorganisms than in natural settings with higher plants or 
430 insects (Gompert \& Messina, 2016; Bono et al., 2017; Wadgymar et al., 2017; Tusso et

$431 a l ., 2021)$. This is likely due to the control of selective agents in experiments as

432 opposed to natural sites where both selective agents and traits under selection may vary

433 (Wadgymar et al., 2017). In our system, high-elevation sites of S. dioica exhibit high

434 winter mortality in S. latifolia and in hybrids in comparison to S. dioica (Favre et al.,

435 2017) suggesting that frost tolerance or the regulation of carbohydrate storage could be

436 under selection. At lowland S. latifolia sites, in contrast, S. dioica and hybrids suffer

437 higher summer mortality than the native S. latifolia, most likely due to drought exposure

438 (Favre et al., 2017). We therefore find it plausible that phenotypic and genetic targets

439 of selection differ between habitats and this can contribute to the conditional neutrality

440 pattern of effect sizes for fitness components as observed here.

441 Fitness is increased by native alleles and decreased by non-native alleles, but not at the 442 same loci

443 A striking result in our study is the association of allele frequencies in the two species 444 with allelic effects on cumulative flowering, even for loci with very small effects.

445 Alleles with positive effects were rare in the non-native species and common or of 446 intermediate frequency in the native species, especially in the S. latifolia habitat. 447 Alleles with positive effects on cumulative flowering in one habitat were neutral in the 448 alternative habitat and could thus easily spread through both species if not lost by drift 449 (Savolainen et al., 2013; Mee \& Yeaman, 2019). Alleles with negative effects on 450 cumulative flowering, on the other hand, had intermediate to high frequencies in the 451 non-native species but were rare in the native species. Negative-effect alleles also 452 included numerous variants that were rare in both species but these loci are most likely 453 linked to causal loci with unknown allele frequencies rather than being causal loci 454 themselves. Negative-effect alleles are most readily interpreted as deleterious load that 455 is only exposed to selection in the alternative habitat and rare, conditionally deleterious 456 variants are expected to arise frequently (Orr, 2005). Conditionally deleterious loci may 457 experience reduced gene flow due to negative selection in the alternative habitat and 458 thereby increase between-lineage differentiation (Mee \& Yeaman, 2019). However, 459 rare variants with conditionally deleterious effects likely remain rare even in the lineage 460 they arose in and will thus not exhibit high differentiation between lineages. Variants 461 with conditionally neutral effects, as reported here, likely play an important role for 462 adaptation, but they are most certainly under-reported, not only because many of these 
463 variants are rare, but also because studies in alternative and relevant environments are 464 needed to detect them.

\section{Implications for hybrid zones and speciation}

Our results suggest that selection favors hybrids that are genetically similar to the native species. In early-generation hybrids with still large genomic regions of each species, as in our experiment, selection will favor genomic regions that contain positive-effect alleles that were more often derived from the native species. At the same time, selection will disfavor regions with negative-effect alleles that were often derived from the non-native species. Both types of conditionally neutral variants thus act as barriers to gene flow in each habitat. In natural hybrid zones between S. dioica and S. latifolia, intermediate individuals are rare and later-generation hybrids are heavily biased toward individuals that are genetically similar to the native species (Minder et al., 2007; Karrenberg \& Favre, 2008). Our study suggests that this may be due not only to backcrossing with the native species but also to ecological selection in each habitat.

Regarding the evolution of adaptative differentiation, our results suggest adaptation through selection on many loci in each species. Alleles with small positive effects had intermediate to high frequencies in the native species and low frequencies in the nonnative species. This may point to a redundant genetic control of adaptation as also suggested by a QTL study under controlled conditions in our study system (Liu \& Karrenberg, 2018) and by studies in other systems, for example in Drosophila (Barghi et al., 2019). Polygenic architectures of adaptation with redundant effects are expected to be transient in time (Yeaman \& Whitlock, 2011; Yeaman, 2015). Thus, even when ecological differentiation is a likely driver of divergence, as in our study system (Goulson \& Jerrim, 1997; Karrenberg \& Favre, 2008; Karrenberg et al., 2019), this may not necessarily manifest in strong, range-wide genetic differentiation at the underlying loci.

\section{Conclusion}

Overall, our study adds to the understanding of how ecological differentiation can promote reproductive isolation and lineage divergence. We detected a polygenic architecture for a fitness component, cumulative flowering, in the campions Silene dioica and S. latifolia using a reciprocal transplant experiment. Allelic effects were 
494 mostly beneficial or deleterious in one habitat and neutral in the other and native-

495 derived alleles were favored. Conditionally neutral effects may result from differences

496 in selection targets in the two habitat types. Variants with conditionally neutral effects

497 provide hidden variation upon which selection can act and facilitate current and future 498 adaptation. The challenges ahead lie in understanding how within-lineage variation for 499 fitness affecting alleles can be reconciled with the evolution of genome-wide 500 differentiation between species.

\section{Acknowledgements}

503 We thank to Rasmus Janson and Karin Steffen for providing help in the molecular lab.

504 We are grateful for support from the Science for Life Laboratory (SciLifeLab) and the

505 National Genomics Infrastructure, NGI, for massive parallel sequencing. Computations

506 were performed on resources provided by SNIC through the Uppsala Multidisciplinary

507 Center for Advanced Computational Science (UPPMAX) under SNIC projects 2017/7-

508406 and 2019/8-21. This work was funded by a project grants of the Swiss National

509 Science Foundation (SNF, no. 3100A-118221), the Swedish Research Council

510 (Vetenskapsrådet, no. 2012-03622) and the Carl Tryggers foundation to SK, as well as

511 by a grant of the German Science Foundation (Deutsche Forschungsgemeinschaft, 512 project no. FA1117/1-2) to AF.

\section{Author contribution}

514 The study was designed by SK with input by AF and XL. AF conducted the transplant 515 experiment with help from SK, XL performed molecular lab work, XL and SG did 516 bioinformatic analyses, SG performed the association analysis and simulations with 517 help from $\mathrm{AB}$ and SK. SK analyzed phenotypes and produced figures with help from 518 SG. SK and SG wrote the paper with input from all other authors.

\section{Data availability}

520 Double-digest RAD (ddRAD) sequencing data is available on NCBI's Short Read 521 Archive (SRA, SUB8324195 https://www.ncbi.nlm.nih.gov/sra/PRJNA669447). The 
variant call format (VCF) file as well as GEMMA input files will be submitted to dryad (project number to be added).

\section{References}

Ågren J, Oakley CG, Lundemo S, Schemske DW. 2017. Adaptive divergence in flowering time among natural populations of Arabidopsis thaliana: Estimates of selection and QTL mapping. Evolution 71(3): 550-564.

Anderson JT, Lee CR, Mitchell-Olds T. 2014. Strong selection genome-wide enhances fitness trade-offs across environments and episodes of selection. Evolution 68(1): 16-31.

Anderson JT, Willis JH, Mitchell-Olds T. 2011. Evolutionary genetics of plant adaptation. Trends in Genetics 27(7): 258-266.

Baena $\square$ Díaz F, Zemp N, Widmer A. 2019. Insights into the genetic architecture of sexual dimorphism from an interspecific cross between two diverging Silene (Caryophyllaceae) species. Molecular Ecology 28(23): 5052-5067.

Barghi N, Tobler R, Nolte V, Jaksic AM, Mallard F, Otte KA, Dolezal M, Taus T, Kofler R, Schlotterer C. 2019. Genetic redundancy fuels polygenic adaptation in Drosophila. PLoS Biology 17(2): e3000128.

Bates D, Maechler M, Bolker B, Walker S. 2015. Fitting linear mixed-effects models using lme4. Journal of Statistical Software 67: 1-48.

Bono LM, Smith LB, Jr., Pfennig DW, Burch CL. 2017. The emergence of performance trade-offs during local adaptation: insights from experimental evolution. Molecular Ecology 26(7): 1720-1733.

Booker TR, Yeaman S, Whitlock MC. 2021. Global adaptation complicates the interpretation of genome scans for local adaptation. Evolution Letters 5(1): 4-15.

Boyle EA, Li YI, Pritchard JK. 2017. An Expanded View of Complex Traits: From Polygenic to Omnigenic. Cell 169(7): 1177-1186.

Bresadola L, Caseys C, Castiglione S, Buerkle CA, Wegmann D, Lexer C. 2019. Admixture mapping in interspecific Populus hybrids identifies classes of genomic architectures for phytochemical, morphological and growth traits. New Phytologist 223(4): 2076-2089.

Catchen J, Hohenlohe PA, Bassham S, Amores A, Cresko WA. 2013. Stacks: an analysis tool set for population genomics. Molecular Ecology 22: 3124-3140. 
554 Chen S, Zhou Y, Chen Y, Gu J. 2018. Fastp: An ultra-fast all-in-one FASTQ preprocessor. Bioinformatics 34(17): i884-i890.

Comeault AA, Soria-Carrasco V, Gompert Z, Farkas TE, Buerkle CA, Parchman subject to a range of intensities of natural selection in Timema cristinae. The American Naturalist 183(5): 711-727.

Danecek P, Auton A, Abecasis G, Albers CA, Banks E, DePristo MA, Handsaker RE, Lunter G, Marth GT, Sherry ST, et al. 2011. The variant call format and VCFtools. Bioinformatics 27(15): 2156-2158.

Exposito-Alonso M, Burbano HA, Bossdorf O, Nielsen R, Weigel D. 2019. Natural selection on the Arabidopsis thaliana genome in present and future climates. Nature 573(7772): 126-129.

Favre A, Widmer A, Karrenberg S. 2017. Differential adaptation drives ecological speciation in campions (Silene): evidence from a multi-site transplant experiment. New Phytologist 213(3): 1487-1499.

Fournier-Level A, Wilczek AM, Cooper MD, Roe JL, Anderson J, Eaton D, Moyers BT, Petipas RH, Schaeffer RN, Pieper B, et al. 2013. Paths to selection on life history loci in different natural environments across the native range of Arabidopsis thaliana. Molecular Ecology 22(13): 3552-3566.

Friedrich HC 1979. Caryophyllaceae. In: Rechinger KH ed. Illustrierte Flora von Mitteleuropa. Hamburg: Parey.

Garrison E, Marth G. 2012. Haplotype-based variant detection from short-read sequencing. 1-9.

Gompert Z, Egan SP, Barrett RD, Feder JL, Nosil P. 2017. Multilocus approaches for the measurement of selection on correlated genetic loci. Molecular Ecology 26(1): 365-382.

\section{Gompert Z, Jahner JP, Scholl CF, Wilson JS, Lucas LK, Soria-Carrasco V,} Fordyce JA, Nice CC, Buerkle CA, Forister ML. 2015. The evolution of novel host use is unlikely to be constrained by trade-offs or a lack of genetic variation. Molecular Ecology 24(11): 2777-2793.

Gompert Z, Lucas LK, Nice CC, Buerkle CA. 2013. Genome divergence and the genetic architecture of barriers to gene flow between Lycaeides idas and L.melissa. Evolution 67(9): 2498-2514. 
Gompert Z, Messina FJ. 2016. Genomic evidence that resource-based trade-offs limit host-range expansion in a seed beetle. Evolution 70(6): 1249-1264.

Goulson D, Jerrim K. 1997. Maintenance of the species boundary between Silene dioica and S. latifolia (red and white campion). Oikos 79(1): 115-126.

Hall MC, Lowry DB, Willis JH. 2010. Is local adaptation in Mimulus guttatus caused by trade-offs at individual loci? Molecular Ecology 19(13): 2739-2753.

Hothorn T, Hornik K, van de Wiel MA, Zeileis A. 2008. Implementing a class of permutation tests: the coin package. Journal of Statistical Software 28(8): 1-23.

Hu XS, Filatov DA. 2015. The large-X effect in plants: Increased species divergence and reduced gene flow on the Silene X-chromosome. Molecular Ecology 25: 2609-2619.

Karrenberg S, Favre A. 2008. Genetic and ecological differentiation in the hybridizing campions Silene dioica and S. latifolia. Evolution 62(4): 763-773.

Karrenberg S, Liu X, Hallander E, Favre A, Herforth $\square$ Rahmé J, Widmer A. 2019. Ecological divergence plays an important role in strong but complex reproductive isolation in campions (Silene). Evolution 73(2): 245-261.

Kawecki TJ, Ebert D. 2004. Conceptual issues in local adaptation. Ecology Letters 7(12): 1225-1241.

\section{Kokko H, Chaturvedi A, Croll D, Fischer MC, Guillaume F, Karrenberg S, Kerr} B, Rolshausen G, Stapley J. 2017. Can evolution supply what ecology demands? Trends in Ecology and Evolution 32(3): 187-197.

Krasovec M, Chester M, Ridout K, Filatov DA. 2018. The mutation rate and the age of the sex chromosomes in Silene latifolia. Current Biology 28(11): 1832-1838.

Leimu R, Fischer M. 2008. A meta-analysis of local adaptation in plants. PloS One 3(12): e4010. doi:4010.1371/journal.pone.0004010.

Leinonen PH, Remington DL, Leppälä J, Savolainen O. 2013. Genetic basis of local adaptation and flowering time variation in Arabidopsis lyrata. Molecular Ecology 22(3): 709-723.

Lenth RV 2020. emmeans: Estimated marginal means, aka least-squares means. R package version 1.5.3. https://CRAN.R-project.org/package=emmeans.

Li H. 2013. Aligning sequence reads, clone sequences and assembly contigs with BWA-MEM. arXiv 1303.3997.

Lind BM, Friedline CJ, Wegrzyn JL, Maloney PE, Vogler DR, Neale DB, Eckert AJ. 2017. Water availability drives signatures of local adaptation in whitebark 
pine (Pinus albicaulis Engelm.) across fine spatial scales of the Lake Tahoe Basin, USA. Molecular Ecology 26(12): 3168-3185.

Liu X, Glémin S, Karrenberg S. 2020. Evolution of putative barrier loci at an intermediate stage of speciation with gene flow in campions (Silene). Molecular Ecology 29(18): 3511-3525.

Liu X, Karrenberg S. 2018. Genetic architecture of traits associated with reproductive barriers in Silene: Coupling, sex chromosomes and variation. Molecular Ecology 27(19): 3889-3904.

\section{Lowry DB, Lovell JT, Zhang L, Bonnette J, Fay PA, Mitchell RB, Lloyd-Reilley J,} Boe AR, Wu Y, Rouquette FM, Jr., et al. 2019. QTL x environment interactions underlie adaptive divergence in switchgrass across a large latitudinal gradient. Proceeding of the National Academy of Sciences, US A 116(26): 12933-12941.

Mee JA, Yeaman S. 2019. Unpacking conditional neutrality: genomic signatures of selection on conditionally beneficial and conditionally deleterious mutations. American Naturalist 194(4): 529-540.

Minder AM, Rothenbuehler C, Widmer A. 2007. Genetic structure of hybrid zones between Silene latifolia and Silene dioica (Caryophyllaceae): evidence for introgressive hybridization. Molecular Ecology 16(12): 2504-2516.

Mitchell-Olds T, Willis JH, Goldstein DB. 2007. Which evolutionary processes influence natural genetic variation for phenotypic traits? Nature Reviews: Genetics 8(11): 845-856.

Nosil P. 2012. Ecological Speciation. Oxford: Oxford University Press.

O'Leary SJ, Puritz JB, Willis SC, Hollenbeck CM, Portnoy DS. 2018. These aren't the loci you'e looking for: Principles of effective SNP filtering for molecular ecologists. Molecular Ecology 27(16): 3193-3206.

Orr HA. 1998. The population genetics of adaptation: the distribution of factors fixed during adaptive evolution. Evolution 52(4): 935-949.

Orr HA. 2005. The genetic theory of adaptation: a brief history. Nature Reviews Genetics 6(2): 119-127.

Peterson BK, Weber JN, Kay EH, Fisher HS, Hoekstra HE. 2012. Double digest RADseq: an inexpensive method for de novo SNP discovery and genotyping in model and non-model species. PloS One 7(5): e37135. 
654 Purcell S, Neale B, Todd-Brown K, Thomas L, Ferreira MAR, Bender D, Maller J, 655 Sklar P, de Bakker PIW, Daly MJ, et al. 2007. PLINK: a tool set for whole656 genome association and population-based linkage analyses. American Journal of $657 \quad$ Human Genetics 81(3): 559-575.

Puritz JB, Hollenbeck CM, Gold JR. 2014. dDocent: a RADseq, variant-calling pipeline designed for population genomics of non-model organisms. PeerJ 2: e431.

R Core Team. 2020. R: A language and environment for statistical computing. $R$ Foundation for Statistical Computing, Vienna, Austria. https://www.Rproject.org/.

Rockman MV. 2012. The QTN program and the alleles that matter for evolution: All that's gold does not glitter. Evolution 66(1): 1-17.

Savolainen O, Lascoux M, Merila J. 2013. Ecological genomics of local adaptation. Nature Reviews: Genetics 14(11): 807-820.

Selby JP, Willis JH. 2018. Major QTL controls adaptation to serpentine soils in Mimulus guttatus. Molecular Ecology 27(24): 5073-5087.

Tiffin P, Ross-Ibarra J. 2014. Advances and limits of using population genetics to understand local adaptation. Trends in Ecology and Evolution 29(12): 673-680.

Tigano A, Friesen VL. 2016. Genomics of local adaptation with gene flow. Molecular Ecology 25(10): 2144-2164.

\section{Tusso S, Nieuwenhuis BPS, Weissensteiner B, Immler S, Wolf JBW. 2021.} Experimental evolution of adaptive divergence under varying degrees of gene flow. Nature Ecology and Evolution 5(3): 338-349.

\section{Wadgymar SM, Lowry DB, Gould BA, Byron CN, Mactavish RM, Anderson JT.} 2017. Identifying targets and agents of selection: innovative methods to evaluate the processes that contribute to local adaptation. Methods in Ecology and Evolution 8(6): 738-749.

Yeaman S. 2015. Local adaptation by alleles of small effect. The American Naturalist 186(S1): S74-S89.

Yeaman S, Whitlock MC. 2011. The genetic architecture of adaptation under migration-selection balance. Evolution 65(7): 1897-1911.

Zhou X, Carbonetto P, Stephens M. 2013. Polygenic modeling with bayesian sparse linear mixed models. Plos Genetics 9(2): e1003264. 


\section{Supporting Information}

690 Figure S1. Principal components analysis including $\mathrm{F}_{0}$ and $\mathrm{F}_{2}$ generations.

691 Figure S2. Principal components analysis of $F_{2}$ families.

692 Figure S3. Posterior distributions of hyperparameters, S. dioica habitat.

693 Figure S4. Posterior distributions of hyperparameters, S. latifolia habitat.

694 Figure S5. Model-averaged effect sizes $(\bar{\beta})$ in the $S$. dioica habitat, plotted against $\bar{\beta}$ in 695 the S. latifolia habitat.

696 Figure S6. Permutation test comparing between-species allele frequency differences 697 among positive- and negative-effect alleles, based on raw effect size estimates $(\hat{\beta})$.

698 Figure S7. Permutation test comparing between-species allele frequency differences 699 among positive- and negative-effect alleles, based on model-averaged effect size 700 estimates $(\bar{\beta})$.

701 Figure S8. Correlations among simulated effects and raw and model-averaged estimates 702 of effect sizes $(\hat{\beta}$ and $\bar{\beta})$.

704 Table S1. Description of source populations and transplant sites.

705 Table S2. Crossing scheme.

706 Table S3. Individuals used for crosses.

707 Table S4. Sparse-effect loci with posterior inclusion probabilities over 0.01. 


\section{Table}

711

712

713

714

715

716

717

718

719

720

Table 1. Genetic architecture of cumulative flowering, a fitness component, in recombinant $\mathrm{F}_{2}$ hybrids between the campions Silene dioica and S. latifolia in a field transplant experiment at sites of each species; given are medians, means and $90 \%$ equal tail credible intervals (90\% cred. int.) of hyperparameters estimated in Bayesian Sparse Linear Mixed Models (BSLMMs): PVE (proportion variation explained, broad-sense heritability, $H^{2}$ ), $P G E$ (proportion variation explained by measurable effects), $h^{2}$ (narrow-sense heritability, $P V E * P G E$ ) and $n-\gamma$ (number of loci with measurable effects). Posterior distributions of hyperparameters are shown in Supplementary Figures S3 and S4.

\section{Hyperparameter}

PVE $\quad$ PGE $\quad h^{2} \quad n-\gamma$

\section{Silene dioica habitat}

$\begin{array}{lcccc}\text { median } & 0.117 & 0.411 & 0.037 & 11 \\ \text { mean } & 0.136 & 0.434 & 0.060 & 42.2 \\ 90 \% \text { cred. int. } & 0.017-0.321 & 0-0.945 & 0-0.198 & 0-205\end{array}$

Silene latifolia habitat

$\begin{array}{lcccc}\text { median } & 0.096 & 0.411 & 0.029 & 16 \\ \text { mean } & 0.124 & 0.431 & 0.054 & 51.4 \\ \text { 90\% cred. int. } & 0.008-0.337 & 0-0.938 & 0-0.193 & 0-225\end{array}$


(a)

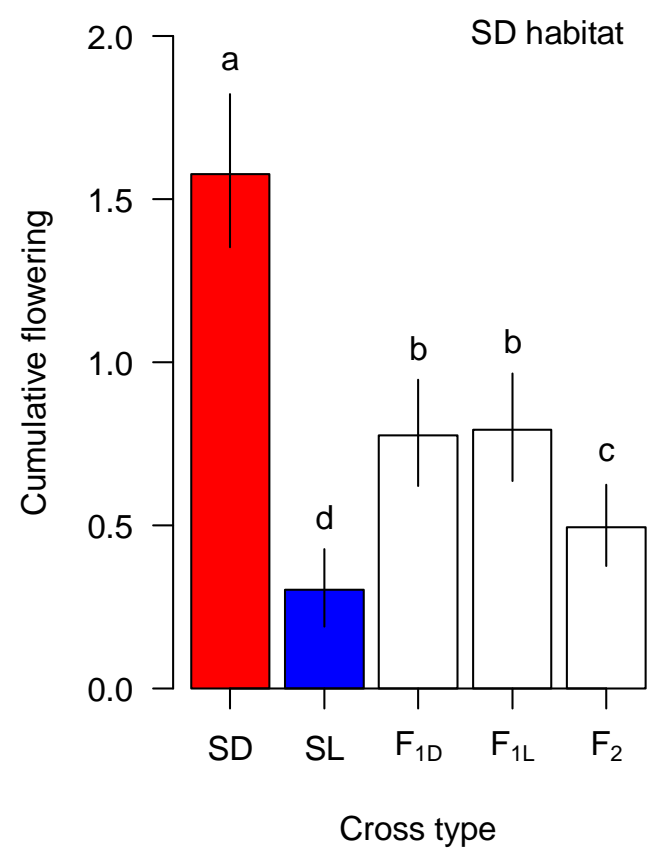

(b)

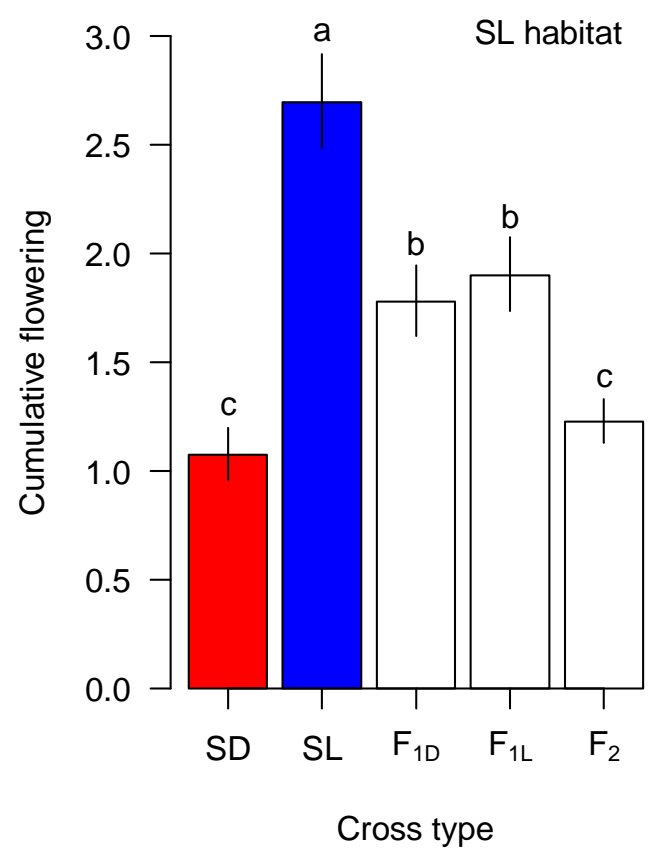

Figure 1. Habitat adaptation in campions (Silene): cumulative flowering (number of times flowered over four years, plus 1 if the plant survived to the end of the experiment), a fitness component, in crosses within each of two campion species, Silene dioica (SD) and S. latifolia (SL), as well as in their first-generation hybrids ( $\mathrm{F}_{1 \mathrm{D}}$ with SD mother, $\mathrm{F}_{1 \mathrm{~L}}, \mathrm{SL}$ mother) and second-generation hybrids $\left(\mathrm{F}_{2}\right)$ in the habitat of each species, (a) SD habitat and (b) SL habitat; least square means and $95 \%$ confidence intervals are given and different letters indicate significant differences between cross types within each habitat. This data is published in Favre et al. (2017 and re-analyzed here for illustration (see text). 
(a)

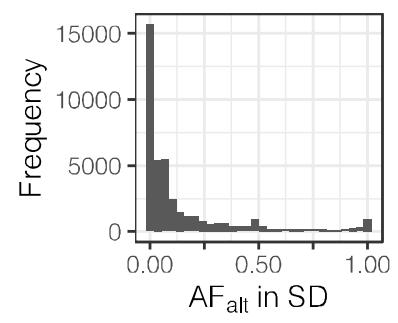

(b)

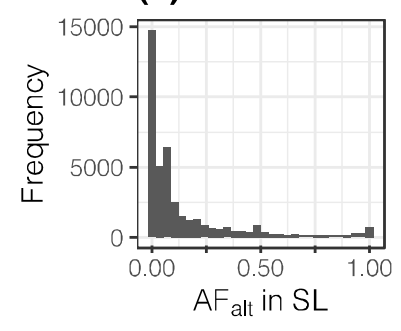

(c)

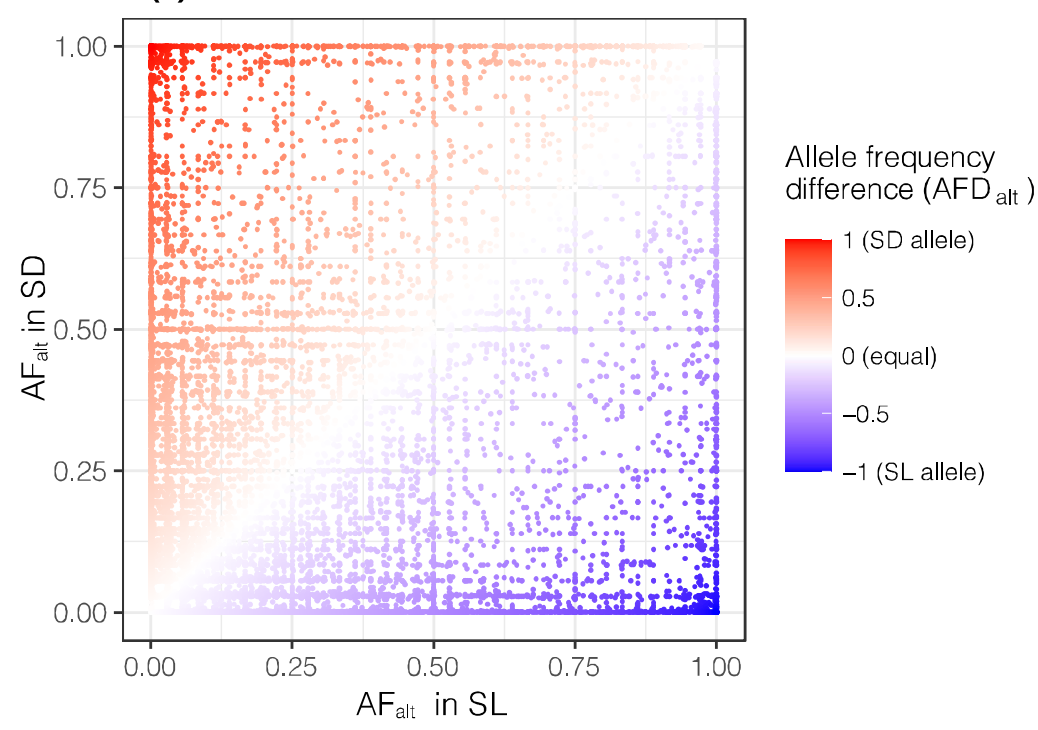

Figure 2. Frequencies of the alternative allele $\left(\mathrm{AF}_{\mathrm{att}}\right)$ in two campion species, Silene dioica

(SD) and S. latifolia (SL) for 42'090 loci used in an association study: (a) distribution of alleles (y-axis) against SL allele frequency ( $\mathrm{x}$-axis) with allele frequency differences $\left(\mathrm{AFD}_{\text {alt }}\right)$ between the two species (SD - SL) indicated as a color gradient from red (allele fixed in SD and absent in SL) to white (equal frequency in both species) to blue (allele fixed in SL and absent in SD). 


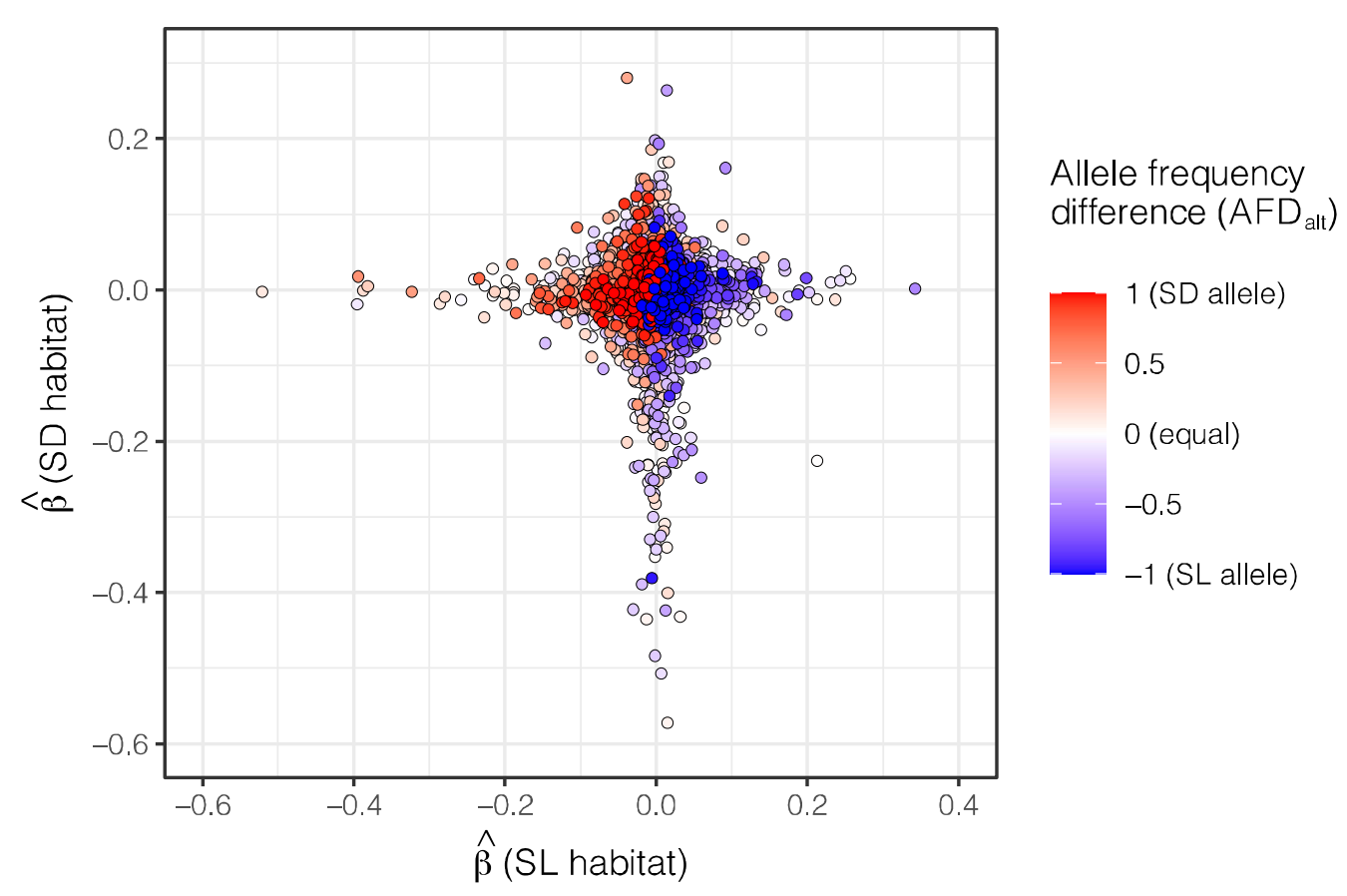

Figure 3. Locus-specific effect sizes of the alternative allele on cumulative flowering, a fitness component, measured in recombinant hybrids between two campion species, Silene dioica (SD) and S. latifolia (SL) that were transplanted into the habitat of each species (SD habitat, y-axis; SL habitat, $\mathrm{x}$-axis). Given are raw effect estimates, $\hat{\beta}$, from Bayesian Sparse Linear Mixed Models (BSLMMs). Allele frequency differences for the alternative allele ( $\left.\mathrm{AFD}_{\text {alt }}\right)$ between the two species (SD-SL) are indicated as a color gradient from blue (allele fixed in SL and absent in $\mathrm{SD})$ to white (equal frequency in both species) to red (allele fixed in SD and absent in SL).

762 Points are plotted in the order of increasing absolute $\mathrm{AFD}_{\text {alt, }}$, such that highly differentiated loci

763 are most visible. Loci with evidence for antagonistic pleiotropy would be have $\hat{\beta}$-values of

764 opposite signs in two habitats (top left, and bottom right), whereas loci consistent with

765 conditional neutrality lie at zero on one axis, but deviate substantially from zero on the other. 
767

768

769

770
FIGURE 4
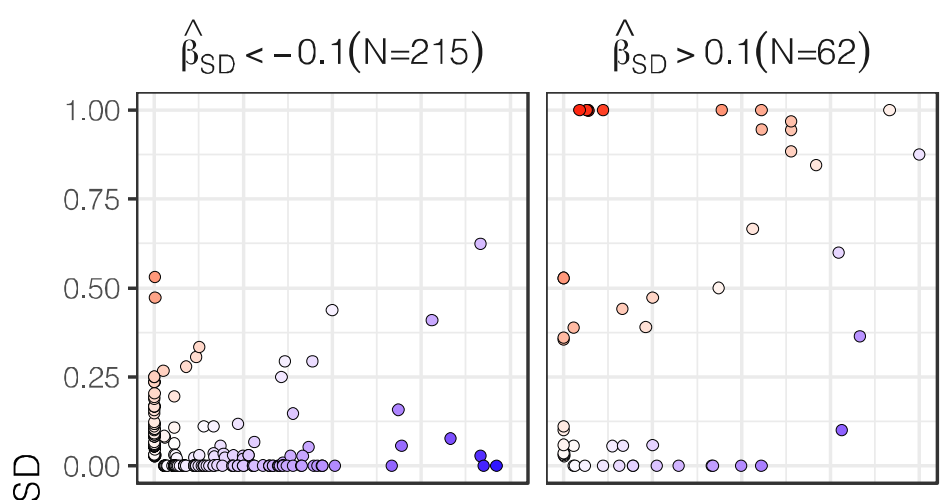

Allele frequency difference (AFD alt $)$
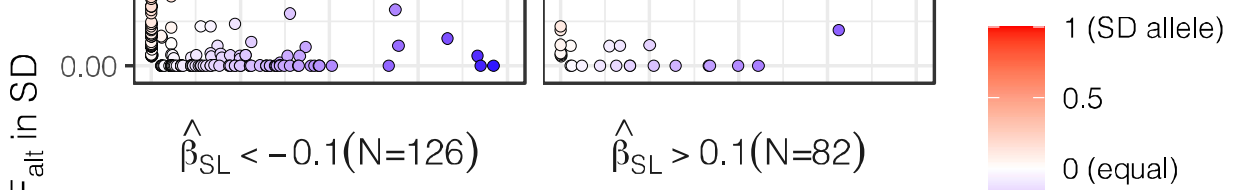

$\hat{\beta}_{\mathrm{SL}}>0.1(\mathrm{~N}=82)$
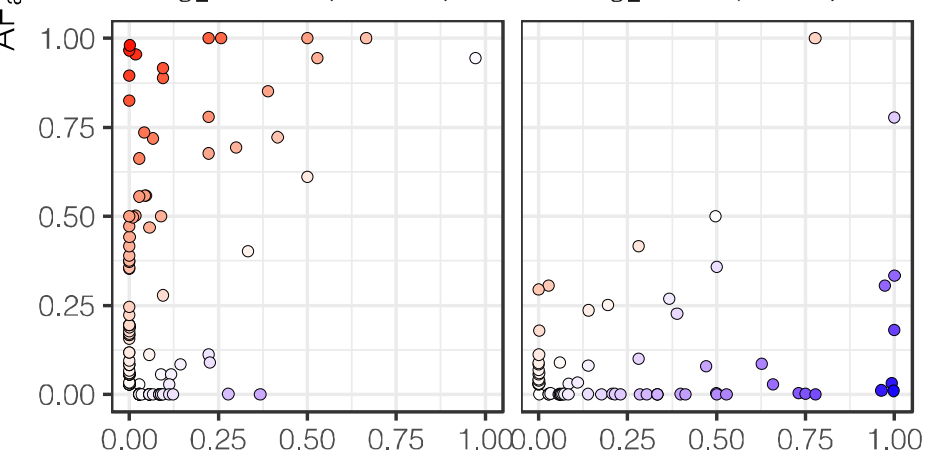

$\mathrm{AF}_{\text {alt }}$ in $\mathrm{SL}$
771

772

773

774

775

776

777

778

779

780

781

782

783

784

785

Figure 4. Frequencies of the alternative allele $\left(\mathrm{AF}_{\mathrm{alt}}\right)$ in two campion species, Silene dioica $(\mathrm{SD}$, $\mathrm{y}$-axis) and S. latifolia (SL, x-axis) for loci associated with cumulative flowering, a fitness component, in the habitat of each species. Shown are loci with absolute raw effect sizes, $|\hat{\beta}|$, over 0.1 (in standard deviation units) in Bayesian Sparse Linear Mixed Models (BSLMMs) with the number of loci $(\mathrm{N}): \hat{\beta}<-0.1$ in the $\mathrm{SD}$ habitat (negative selection), $\hat{\beta}>0.1$ in the $\mathrm{SD}$ habitat (positive selection), $\hat{\beta}<-0.1$ in the SL habitat (negative selection), and $\hat{\beta}>0.1$ in the SL habitat (positive selection). Allele frequency differences for the alternative allele $\left(\mathrm{AFD}_{\text {alt }}\right)$ between the two species (SD-SL) are indicated as a color gradient from blue (allele fixed in SL and absent in $\mathrm{SD})$ to white (equal frequency in both species) to red (allele fixed in SD and absent in SL). Positive selection is associated with native alleles, whereas alleles under negative selection are more common in the non-native species (see also text and Supporting Information Figs. S6 and S7). 
(a)

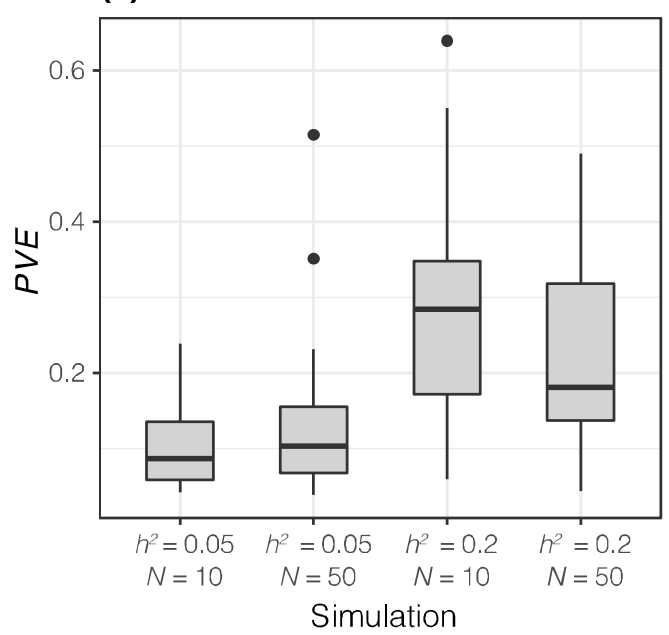

(c)

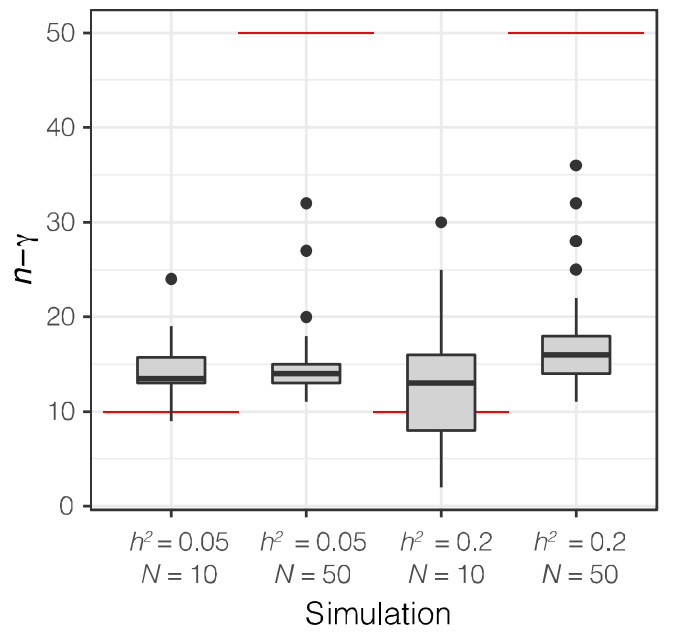

(b)

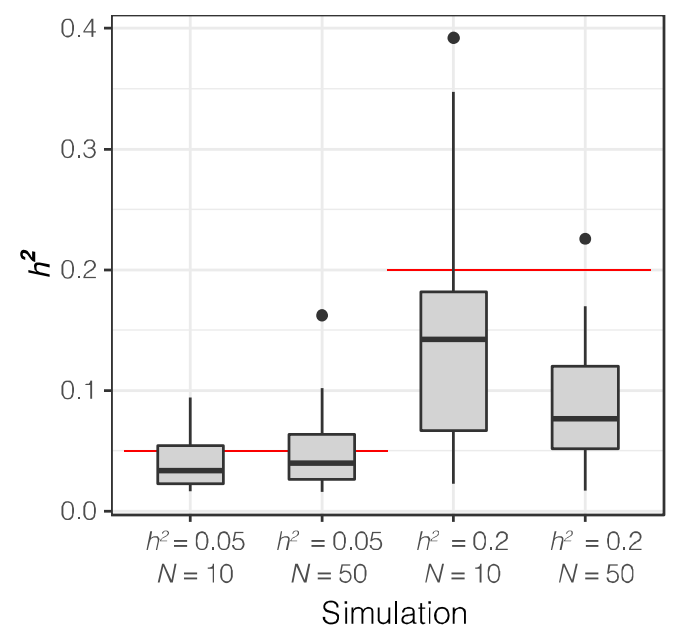

(d)

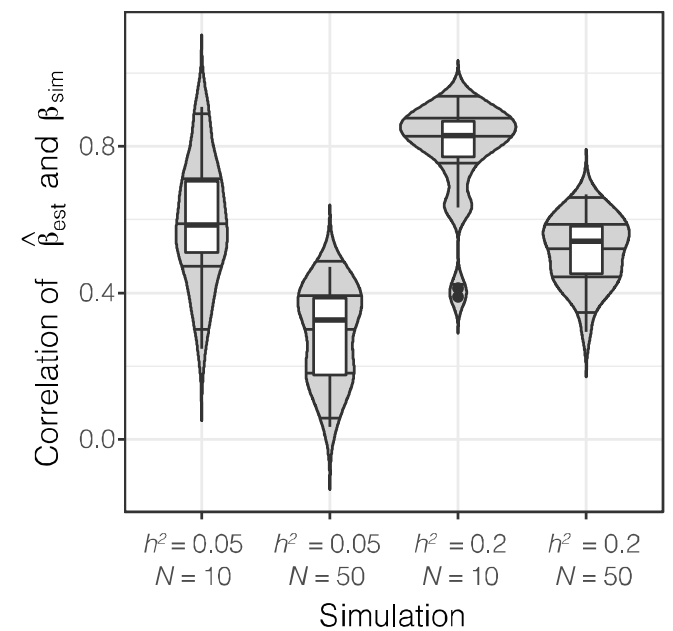

Figure 5. Performance analysis of Bayesian Sparse Linear Mixed Models (BSLMM) on simulated data based on a dataset from campions (Silene, 150 individuals, 42 '090 loci).

791 Simulated phenotypes had narrow-sense heritabilities $\left(h^{2}\right)$ of 0.05 or 0.20 with 10 or 50 792 functional loci $(N) ; 30$ replicate simulations per scenario were used and boxplots or 793 violin plots summarize variation in medians of posterior distributions of 794 hyperparameters among simulation replicates as well as of correlations of simulated and estimated per-locus effect sizes. (a) percent variation explained ( $P V E$, interpreted as broad-sense heritability, $H^{2}$ ), (b) narrow-sense heritability $h^{2}$ (as $P V E * P G E[P G E$,

797 proportion of sparse effects in $P V E]$ ), (c) number of sparse-effect loci $(n-\gamma)$ and (d), correlation of raw estimated effect sizes for individual loci $\hat{\beta}_{\text {est }}$ with simulated effect sizes $\beta_{\text {sim. }}$. On (b) and (c) simulated values are indicated with red lines. 